\title{
Sini San ameliorates duodenal mucosal barrier injury and low-grade inflammation via the CRF pathway in a rat model of functional dyspepsia
}

\author{
CHUNYANG ZHU, LUQING ZHAO, JINGYI ZHAO and SHENGSHENG ZHANG \\ Digestive Disease Center, Beijing Hospital of Traditional Chinese Medicine, \\ Capital Medical University, Beijing 100010, P.R. China
}

Received June 7, 2019; Accepted September 19, 2019

DOI: $10.3892 /$ ijmm.2019.4394

\begin{abstract}
The gut-brain interaction is associated with impaired duodenal mucosal integrity and low-grade inflammation, which have been proven to be important pathological mechanisms of functional dyspepsia (FD). Sini San (SNS) is a classical Chinese medicine used to treat FD, but its underlying mechanisms are poorly understood. The aim of the present study was to evaluate the effects of SNS on duodenal mucosal barrier injury and low-grade inflammation with $\mathrm{FD}$, and to assess its potential molecular mechanisms on the brain-gut axis. FD rats were established using the iodoacetamide and tail-squeezed methods. The expression of corticotropin-releasing factor (CRF), CRF receptor 1 (CRF-R1) and CRF-R2, were determined by western blot analysis and/or immunohistochemistry (IHC). In addition, mast cell (MC) migration was assessed by IHC with an anti-tryptase antibody, and histamine concentration was quantified using ELISA. The mRNA expression levels of tryptase and protease-activated receptor 2 (PAR-2) were quantified using reverse transcription-quantitative PCR, and the protein expression levels of zona occludens protein 1 (ZO-1), junctional adhesion molecule 1 (JAM-1), $\beta$-catenin and E-cadherin were determined via western blot analysis. It was demonstrated that the expression level of CRF was downregulated in the central nervous system and duodenum following SNS treatment,
\end{abstract}

Correspondence to: Dr Shengsheng Zhang, Digestive Disease Center, Beijing Hospital of Traditional Chinese Medicine, Capital Medical University, 23 Meishuguanhou Street, Beijing 100010, P.R. China

E-mail: zhss2000@163.com

Abbreviations: FD, functional dyspepsia; SNS, Sini San; CRF, corticotropin-releasing factor; CRF-R, corticotropin-releasing factor receptor; PAR-2, protease-activated receptor 2; ZO-1, zona occludens protein 1; JAM-1, junctional adhesion molecule 1

Key words: Sini san, functional dyspepsia, corticotropin-releasing factor, mucosal barrier integrity, low-grade inflammation, mast cell and that SNS modulated the expression of both CRF-R1 and CRF-R2. In addition, SNS suppressed MC infiltration and the activity of the tryptase/PAR-2 pathway in the duodenum. Furthermore, treatment with SNS restored the normal expression levels of ZO-1, JAM-1 and $\beta$-catenin in FD rats. These findings suggested that the therapeutic effects of SNS on FD were achieved by restoring mucosal barrier integrity and suppressing low-grade inflammation in the duodenum, which was at least partially mediated via the CRF signaling pathway.

\section{Introduction}

Functional dyspepsia (FD) is a common functional gastrointestinal disorder marked chiefly by postprandial fullness, early satiation, epigastric pain and epigastric burning (1). The pathogenic mechanisms of FD are complex, and include gastric emptying, impaired gastric accommodation, duodenal inflammation, mucosal permeability and psychosocial factors (2). The role of the duodenum in the pathogenesis of FD is well known, and the integrity of the duodenal mucosal barrier is significant to the maintenance of normal duodenal function (3). Previous findings have shown that impaired intestinal barrier function and low-grade inflammation are apparent in the duodenum of patients with FD (4,5).

Interactions between the brain and the gut serve an important role in functional gastrointestinal diseases (FGIDs), including FD, and there is evidence to suggest that the central nervous system and gut interact bi-directionally in FD patients (6). Corticotropin-releasing factor (CRF) is one of the brain peptides that co-ordinates the behavioral, endocrine, autonomic and visceral responses to stress (7). Research indicates that the binding of CRF to its receptors, primarily CRF receptor 1 (CRF-R1) and CRF-R2, results in mast cell (MC) degranulation, which contributes to the characteristic low-grade inflammation observed in FD patients (8). MCs are one of the key contributing factors to duodenal micro-inflammation, which can further influence epithelial barrier integrity via the tryptase/proteinase-activated receptor 2 (PAR-2) pathway $(9,10)$.

Sini San (SNS) is a traditional Chinese medicine that has been used for thousands of years. In the clinic, SNS is widely used to treat FGIDs, particularly FD $(11,12)$. SNS is 
composed of an equal ratio of four different herbs: i) Chaihu (Radix Bupleuri Chinensis); ii) Baishao (Radix Paeoniae Alba); iii) Zhishi (Fructus Aurantii Immaturus); and iv) and Gancao (Radix Glycyrrhizae). It has been confirmed that SNS can improve depression by regulating the expression of central neurotransmitters (13), and improve duodenal tight junction integrity in FD rats (14). However, the protective mechanism of SNS on low-grade inflammation and duodenal mucosal barrier integrity have not been extensively researched.

The aim of the present study was to determine whether SNS restores mucosal barrier integrity and suppresses low-grade inflammation in the duodenum via the CRF signaling pathway.

\section{Materials and methods}

SNS preparation. SNS, which consisted of Chaihu (Radix Bupleuri Chinensis), Baishao (Radix Paeoniae Alba), Zhishi (Fructus Aurantii Immaturus) and Gancao (Radix Glycyrrhizae) was purchased from Beijing Xinglin Pharmaceutical Industry Co., Ltd., and identified as eligible medicinal material according to the Pharmacopoeia of the People's Republic of China (2015 edition) (15). SNS was decocted by the Beijing Hospital of Traditional Chinese Medicine, Capital Medical University (Beijing, China). Briefly, the raw herbs were combined to a total weight of $200 \mathrm{~g}$ and impregnated in 1,000 $\mathrm{ml}$ distilled water for $30 \mathrm{~min}$ at room temperature. After boiling for $30 \mathrm{~min}, 200 \mathrm{ml} \mathrm{SNS}$ was harvested and set aside. Another 200-ml distilled water was added to the original herb mixture, which was boiled for a further $30 \mathrm{~min}$, and both decoctions were combined to generate a 400-ml SNS preparation at a concentration of $0.5 \mathrm{~g} / \mathrm{ml}$. The preparation was filtered through a 100 -mesh sieve to remove particulate material.

Animals. A total of 36, 7-day-old (12-15 g), male Sprague Dawley rats were purchased from SPF (Beijing) Biotechnology Co., Ltd. The rats were housed in a standardized environment with a 12-h light/dark cycle, at a temperature of $20-26^{\circ} \mathrm{C}$ (humidity, $50 \pm 5 \%$ ) and free access to food and water. In the present study, the iodoacetamide (IA)-treated and tail-squeezed methods were combined to generate a modified rat model of functional dyspepsia that better simulated pathogenesis of FD patients. Previous findings revealed that the combined model rats exhibited obvious symptoms of FD, such as the reduction of gastric accommodation and gastric emptying (16). After acclimation for 3 days prior to the experiment, 10-day-old rats were randomly divided into 3 groups of 12 rats each: The control, model and SNS groups. The FD model rats received $0.2 \mathrm{ml} 0.1 \%$ IA in $2 \%$ sucrose by oral gavage, every day for 6 days, and the control group received an equal volume of $2 \%$ sucrose only. At 7 weeks old, tail-clamping was conducted on the FD rats for a further 7 days, and surgical forceps were used to clamp the distal third of the tail, every $4 \mathrm{~h}$ for $30 \mathrm{~min}, 3$ times per day. Then, the FD rats were randomly divided into two groups that received intragastric administration of SNS (1 ml/100 g) or an equal volume of water as a vehicle control, for 7 consecutive days. The rats received euthanasia by intraperitoneal injection of sodium pentobarbital (100 $\mathrm{mg} / \mathrm{kg}$ of body weight).
The present study was performed in accordance with the Guide for the Care and Use of Laboratory Animals published by the National Institutes of Health, and was approved by the Animal Care and Use Committee of Beijing Institute of Traditional Chinese Medicine (no. 2018030101).

Western blot analysis. Rats were decapitated for hypothalamus and duodenum tissues after euthanasia, and samples were fixed immediately in liquid nitrogen, and stored at $-80^{\circ} \mathrm{C}$ in ultra-low temperature freezer. The hypothalamus and duodenum tissues were homogenized with an appropriate volume of RIPA buffer with protease inhibitor cocktail and $1 \mathrm{mM}$ PMSF (Beijing Solarbio Science \& Technology Co., Ltd.). The protein concentration was determined using a bicinchoninic acid (BCA) assay kit and the protein samples were mixed with $5 \mathrm{X}$ loading buffer. Denatured protein ( $80 \mu \mathrm{g} / \mathrm{lane}$ ) was separated using $10 \%$ SDSPAGE and transferred to PVDF membranes. After blocking with nonfat milk in TBST $(10 \mathrm{mmol} / \mathrm{l}$ Tris- $\mathrm{HCl}$, $0.5 \mathrm{ml} / 1$ Tween-20 and $0.15 \mathrm{~mol} / 1 \mathrm{NaCl}, \mathrm{pH} \mathrm{7.2)}$ for $1 \mathrm{~h}$ at the room temperature, the membranes were incubated at $4^{\circ} \mathrm{C}$ overnight (about $16 \mathrm{~h}$ ) with the following primary antibodies: Anti-CRF (1:500; 10944-1-AP, ProteinTech Group, Inc.), anti-CRF-R1 (1:1,000; 20967-1-AP, ProteinTech Group, Inc.), anti-CRF-R2 (1:1,000; CSB-PA623797LA01HU, Cusabio Technology LLC), anti-ZO-1 (1:1,000; 21773-1-AP,ProteinTech Group, Inc.), anti-JAM-1 (1:500; BS-3651R, BIOSS), anti- $\beta$-catenin (1:1,000; 51067-2-AP, ProteinTech Group, Inc.), anti-E-cadherin $(1: 1,000 ; 20874-1-A P$, ProteinTech Group, Inc.) and anti- $\beta$-actin (1:10,000; GB12001, Wuhan Servicebio Technology Co., Ltd.). After washing three times with TBST, the membranes were incubated with the following secondary antibodies: Goat anti-rabbit or goat anti-mouse IgG (1:10,000; 072-06-15-06 and 072-07-18-06, KPL, Inc.). The protein levels were quantified as relative grey value using ImageJ software v1.51 (National Institutes of Health).

ELISA. The duodenal tissues were homogenized and the supernatants were collected. After quantifying the total protein concentration using a BCA assay kit, the concentrations of histamine were determined with a histamine ELISA kit (E-EL-0032c, Elabscience Biotechnology Co., Ltd.) according to the manufacturer's instructions.

Reverse transcription-quantitative $(R T-q) P C R$. Total RNA was extracted from the duodenal tissue using TRIzol ${ }^{\circledR}$ reagent (Thermo Fisher Scientific, Inc.), and $5 \mu \mathrm{g}$ total RNA was reverse-transcribed into cDNA. The gene expression levels of tryptase and PAR-2 were determined using the SYBR ${ }^{\circledR}$ Master Mix (Promega Corporation) and the CFX 96 Real-time PCR System (Bio-Rad Laboratories, Inc.), and individual gene expression levels were normalized to those of $\beta$-actin. The thermocycling conditions were as follows: An initial step at $95^{\circ} \mathrm{C}$ for $2 \mathrm{~min}, 40$ cycles of denaturation at $95^{\circ} \mathrm{C}$ for $15 \mathrm{sec}$, and annealing/extension at $60^{\circ} \mathrm{C}$ for $1 \mathrm{~min}$. The primer sequences used were: Tryptase forward, 5'-GCTTCCATC CAGTACCGC-3', and reverse, 5'-ACCTTTGCAGATCAA AGG-3'; PAR-2 forward, 5'-TTGGCAGACCTCCTCTCTGT-3', and reverse, 5'-GAGGCAGGTCATGAAAAGGA-3'; $\beta$-actin forward, 5'-AGTTGCGTTACACCCTTTC-3', and reverse, 5'-CACCTTCACCGTTCCAGT-3'. 
Immunohistochemistry (IHC). Duodenal and spinal cord (T8-T10) sections were fixed in $4 \%$ paraformaldehyde at room temperature, embedded in paraffin, cut into sections $(4-\mu \mathrm{m})$, and mounted on glass slides. The sections were heated to $60^{\circ} \mathrm{C}$ for $20 \mathrm{~min}$, deparaffinized in xylene and dehydrated in alcohol. Antigen retrieval was performed in a pressure cooker using sodium citrate buffer ( $\mathrm{pH}$ 6.0). The sections were then soaked in $3 \% \mathrm{H}_{2} \mathrm{O}_{2} /$ methanol solution to inhibit endogenous peroxidase activity, and then blocked with $3 \%$ BSA. The sections were incubated overnight at $4^{\circ} \mathrm{C}$ with anti-CRF (1:100; 10944-1-AP, ProteinTech Group, Inc.), and anti-tryptase (1:100; ab2378, Abcam) primary antibodies. After washing in PBS ( $\mathrm{pH} 7.4$ ), the sections were incubated with the following secondary antibodies: Horseradish peroxidase-labeled goat anti-rabbit or goat anti-mouse IgG (1:200; GB2301 and GB23303, Wuhan Servicebio Technology Co., Ltd.). The tissues were then incubated with $0.05 \%$ 3,3'-diaminobenzidine tetrachloride for colorimetric development, and subsequently counterstained with hematoxylin prior to dehydration with ethanol and xylene. Images were obtained using a light microscope of 100-200 times multiplication (Carl Zeiss AG), and the immunoreactivity of CRF and MC tryptase was determined using the Image Pro Plus 6.0 image analysis software system (Media Cybernetics, Inc.).

Statistical analysis. Statistical analyses were performed using SPSS 20.0 software (IBM Corp). Data are expressed as the mean \pm SEM. One-way analysis of variance (ANOVA) followed by the least significant difference post hoc test was used to determine the statistical significance among the groups. $\mathrm{P}<0.05$ was considered to indicate a statistically significant difference.

\section{Results}

SNS reduces the expression levels of CRF in the duodenum and central nervous system. The CRF signaling pathway is an important component of the brain-gut interaction, coordinating visceral responses to stress (17). We aimed to determine whether a CRF-related pathway was involved in the response to SNS treatment in FD. The central and peripheral CRF expression levels were determined using western blotting (Fig. 1A) and/or IHC (Fig. 2). The results showed that the protein expression level of CRF was markedly increased in the hypothalamus (Fig. 1B) and duodenum (Fig. 1C) of FD rats, compared with the control group (hypothalamus: $0.38 \pm 0.01$ vs. $0.09 \pm 0.01 ; \mathrm{P}<0.01$ ) (duodenum: $0.27 \pm 0.02$ vs. $0.11 \pm 0.02, \mathrm{P}<0.01)$. SNS treatment ameliorated the expression levels of CRF in both the hypothalamus and the duodenum (hypothalamus: $0.18 \pm 0.02$ vs. $0.38 \pm 0.01, \mathrm{P}<0.01$ ) (duodenum: $0.19 \pm 0.02$ vs. $0.27 \pm 0.02, \mathrm{P}<0.01$ ). A similar expression trend was observed in spinal cord tissues. The protein expression levels of CRF in spinal cord (T8-T10) (Fig. 2A) and duodenal tissues (Fig. 2B) were also detected, using IHC. The average optical density value (IOD/area) increased in spinal cord and duodenum of model group (both $\mathrm{P}<0.01$ ), but was decreased following SNS treatment (both $\mathrm{P}<0.01$ ).

$S N S$ regulates the expression of $C R F-R 1$ and $C R F-R 2$ in the duodenum. CRF activity is initiated via the activation of CRF receptors, primarily CRF-R1 and CRF-R2, within the gut wall (18). Therefore, the expression of CRF-R1 and CRF-R2 was detected in duodenal tissues (Fig. 3A). The expression levels of CRF-R1 (Fig. 3B) increased markedly in the FD group compared with the control group $(0.17 \pm 0.02$ vs. $0.08 \pm 0.01$; $\mathrm{P}<0.01)$, but were reduced in the SNS group (0.08 \pm 0.01 vs. $0.17 \pm 0.02 ; \mathrm{P}<0.01)$. However, the expression level of CRF-R2 (Fig. 3C) was the opposite of that of CRF-R1 (FD group vs. control group, $0.12 \pm 0.02$ vs. $0.17 \pm 0.02 \mathrm{P}<0.01$; SNS group vs. FD group, $0.14 \pm 0.01$ vs. $0.12 \pm 0.02 ; \mathrm{P}<0.05)$. These findings suggested that SNS downregulated the expression level of CRF in the duodenum and central nervous system, and modulated the expression of both CRF-R1 and CRF-R2.

SNS modulates MC infiltration. MCs are an important contributor to the mucosal micro-inflammation observed in FD (19), and the fact that MC activation negatively impacts intestinal permeability is well known $(4,20,21)$. Histamine and tryptase are important indicators of MC activation, thus the influence of SNS on MC infiltration was assessed by IHC staining of tryptase (Fig. 4A) and the histamine concentration quantified using ELISA (Fig. 4B). The results indicated a prominent increase in the average optical density of tryptase-positive cells in FD rats, compared with that of the control rats $\left[(6.27 \pm 0.54) \times 10^{3}\right.$ vs. $\left.(1.57 \pm 0.26) \times 10^{3} ; \mathrm{P}<0.01\right]$. Furthermore, the average optical density of tryptase-positive cells was decreased following SNS treatment $\left[(2.30 \pm 0.32) \times 10^{3}\right.$ vs. $\left.(6.27 \pm 0.54) \times 10^{3} ; \mathrm{P}<0.01\right]$. The ELISA results showed that the concentration of histamine was significantly higher in the FD rats, compared with the control group $(3.54 \pm 0.33$ vs. $1.42 \pm 0.24 ; \mathrm{P}<0.01)$, which was suppressed in response to SNS treatment $(2.06 \pm 0.25$ vs. $3.54 \pm 0.33 ; \mathrm{P}<0.01)$. These data demonstrate that SNS suppressed low-grade inflammation by alleviating MC infiltration.

SNS decreases the mRNA expression level of tryptase and $P A R-2$. PAR-2 regulates gastrointestinal motility and epithelial barrier function (22), and is activated by proteases (principally MC tryptase) in the intestine (23). The potential effects of SNS on MC-associated tryptase/PAR-2 in the duodenum of FD rats were therefore determined. Tryptase (Fig. 5A) and PAR-2 (Fig. 5B) mRNA expression levels were significantly higher in the duodenum of FD rats than in the control group (PAR-2: $2.22 \pm 0.36$ vs. $1.00 \pm 0.15, \mathrm{P}<0.01$; tryptase: $3.66 \pm 0.46$ vs. $1.15 \pm 0.14, \mathrm{P}<0.01)$. Following SNS treatment in the FD group, the mRNA expression levels of PAR-2 (1.11 \pm 0.18 vs. $2.22 \pm 0.36$; $\mathrm{P}<0.01)$ and tryptase $(1.30 \pm 0.17$ vs. $3.66 \pm 0.46 ; \mathrm{P}<0.01)$ were significantly reduced. These findings suggest that the MC tryptase/PAR-2 signaling pathway can be regulated by SNS.

SNS restores duodenal mucosal barrier function in FD rats. Impaired duodenal mucosal integrity is apparent in patients with FD, and the symptoms of FD are attributable to this phenomenon. Thus the present study also aimed to determine whether SNS restores the duodenal mucosal barrier by regulating the expression of tight junction (TJ) and adherens junction (AJ) proteins (Fig. 6A and D). The protein expression levels of $\mathrm{ZO}-1$ (Fig. 6B; $0.22 \pm 0.02$ vs. $0.51 \pm 0.02$; $\mathrm{P}<0.01$ ), JAM-1 (Fig. 6C; $0.16 \pm 0.02$ vs. $0.24 \pm 0.03 ; \mathrm{P}<0.01$ ) and $\beta$-catenin (Fig. $6 \mathrm{E}$; $0.21 \pm 0.04$ vs. $0.39 \pm 0.05 ; \mathrm{P}<0.01)$ were significantly reduced in the duodenal tissues of FD rats, compared with those of the control group. Furthermore, SNS treatment enhanced the 

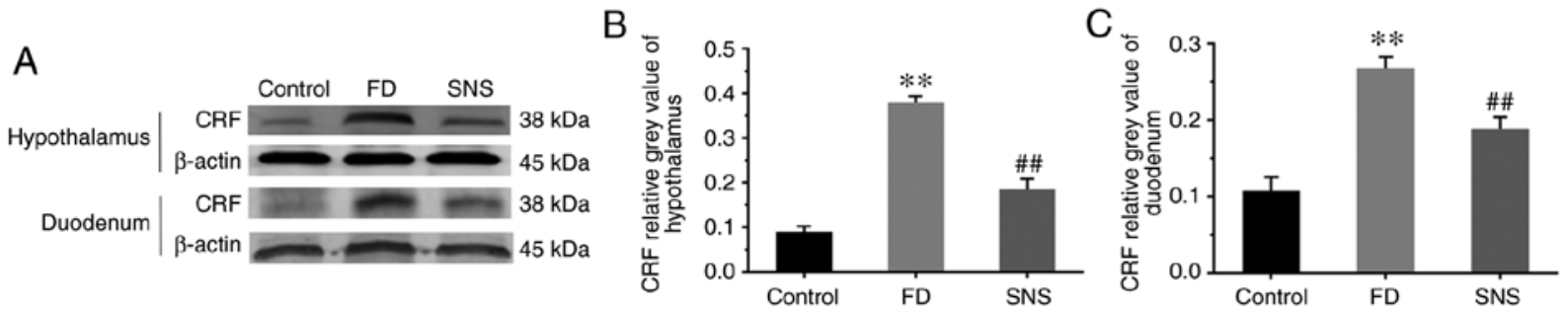

Figure 1. (A) Western blot analysis of CRF in the hypothalamus and duodenum. The relative grey value of CRF in tissues of the (B) hypothalamus and (C) duodenum. Expression levels of CRF were elevated in the hypothalamus and duodenum. SNS reduced the expression level of CRF in these two tissues. $\mathrm{N}=6 ;{ }^{* *} \mathrm{P}<0.01$ compared with the control group; ${ }^{\# \#} \mathrm{P}<0.01$ compared with the model group. CRF, corticotropin-releasing factor; SNS, Sini San.

A

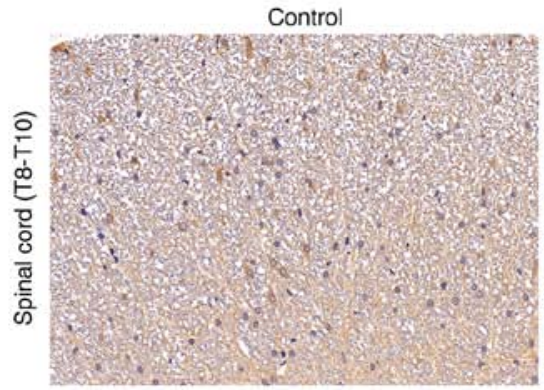

B

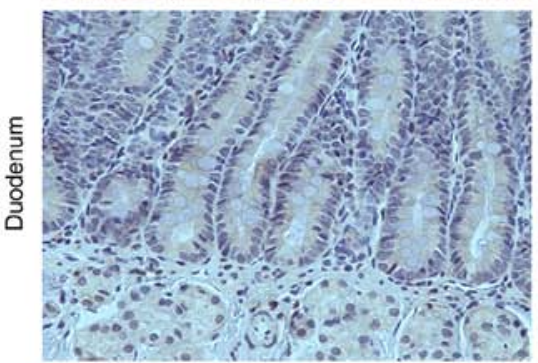

FD
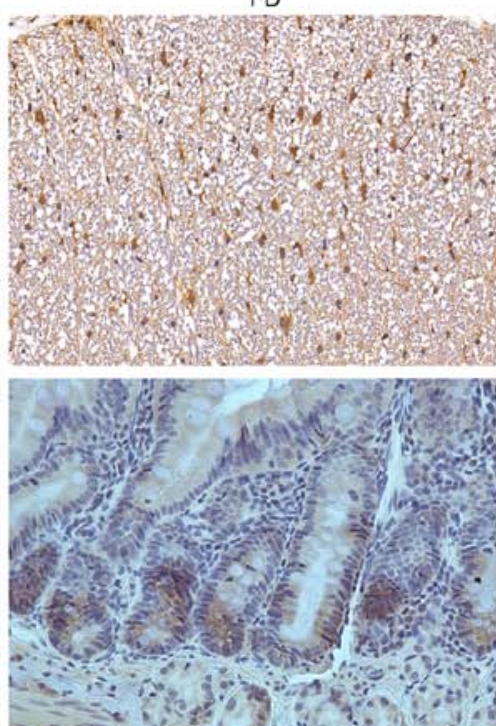
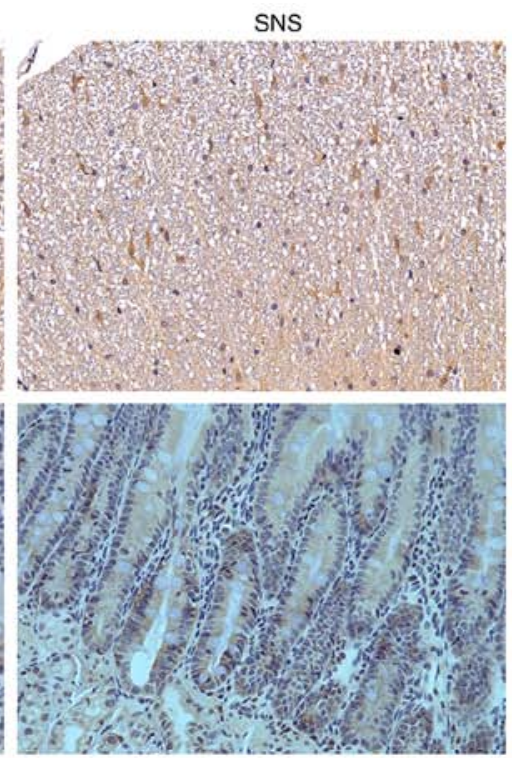

Figure 2. Immunohistochemical staining of CRF in the spinal cord and duodenum. Expression levels of CRF were increased in the (A) spinal cord and (B) the duodenum. SNS reduced the expression level of CRF in these tissues. Magnification, x200; N=4. CRF, corticotropin-releasing factor; SNS, Sini San.

expression levels of these proteins in FD rats $(\mathrm{ZO}-1: 0.47 \pm 0.01$ vs. $0.22 \pm 0.02, \mathrm{P}<0.01$; JAM-1: $0.19 \pm 0.03$ vs. $0.16 \pm 0.02, \mathrm{P}<0.05$; and $\beta$-catenin: $0.37 \pm 0.04$ vs. $0.21 \pm 0.04, \mathrm{P}<0.01)$. However, there were no marked abnormalities in the protein expression levels of E-cadherin among the three groups (Fig. 6F; control group vs. FD group: $\mathrm{P}=0.868$; FD group vs. $\mathrm{SNS}$ group: $\mathrm{P}=0.624$ ). These results indicated that SNS upregulated the expression of ZO-1, JAM-1 and $\beta$-catenin, and that it may potentially be used to restore the duodenal mucosal barrier.

\section{Discussion}

The present study demonstrated that SNS treatment restored duodenal mucosal barrier integrity and ameliorated low-grade inflammation by modulating the gut-brain interaction in a rat model of FD. The underlying therapeutic mechanisms of SNS are the regulation of the CRF pathway and suppressed infiltration of MCs in the duodenum, and a subsequent increase the expression levels of ZO-1, JAM-1 and $\beta$-catenin.

The integrity of the duodenal mucosal barrier is of great significance to keep the intestinal homeostasis (24). Changes in mucosal permeability and tight junction protein expression have been reported in numerous gastrointestinal diseases, including ulcerative colitis, Crohn's disease and irritable bowel syndrome (25-27). Previous studies have found that intestinal permeability is increased in patients with $\operatorname{FD}(4,20)$, and that the intestinal epithelium between the enterocytes is sealed by tight and adherens junctions, and desmosomes (28). As important components of the epithelial mechanical barrier, TJ proteins protect the intestinal mucosa from foreign antigens, toxins and invasion by environmental microorganisms (29). ZO-1 is a scaffolding protein that plays a key role in the formation of TJs (30), while JAM-1 is localized to the TJs of epithelial and endothelial cells and is closely associated with the regulation of junctional integrity and permeability (31). Research has suggested that acute stress reduces the expression of TJ proteins in the duodenal mucosa (32). $\beta$-catenin and E-cadherin are important components of cell-to-cell adhesion proteins, which maintain cell and tissue polarity and integrity (33). Previous research demonstrated that SNS reduced duodenum mucosal permeability and restored the expression levels of claudin-1 and occluding (14), but it remained unclear whether SNS could regulate AJ proteins, or indeed, other TJ proteins. In the present study, decreased expression levels of ZO-1, JAM-1 and $\beta$-catenin were evident in FD rats, compared with the control group, and SNS treatment restored these expression levels to a significant degree. Another key finding of the present study was that SNS treatment also restored duodenal epithelial barrier 

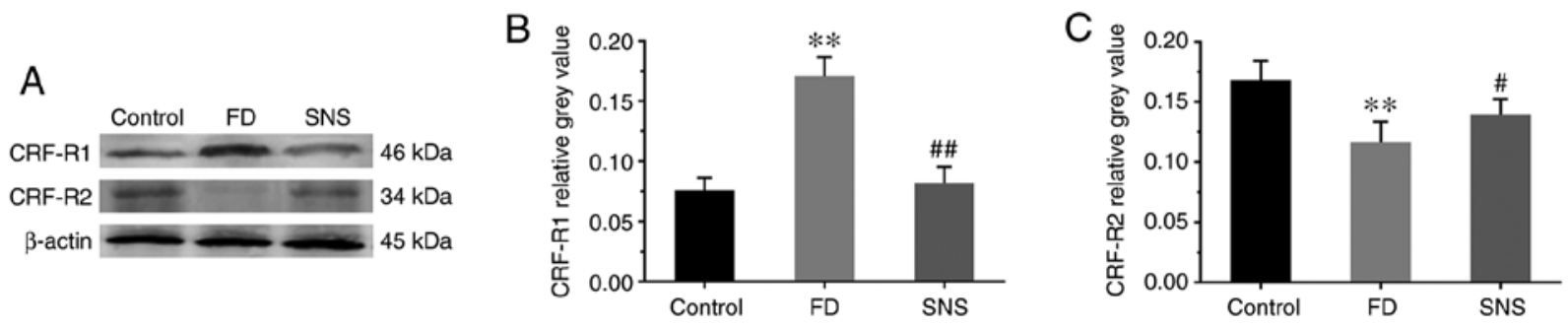

Figure 3. (A) Western blot analysis of CRF-R1 and CRF-R2 in the duodenum. Relative grey value of (B) CRF-R1 and (C) CRF-R2 expression levels in duodenal tissues. Expression levels of CRF-R1 were elevated in FD rats and reduced in the SNS group. Expression patterns of CRF-R2 were the reverse of those for CRF-R1. $\mathrm{N}=6 .{ }^{* *} \mathrm{P}<0.01$ compared with the control group; ${ }^{*} \mathrm{P}<0.05$ and ${ }^{\# \#} \mathrm{P}<0.01$ compared with the model group. $\mathrm{CRF}$, corticotropin-releasing factor; CRF-R1, corticotropin-releasing factor receptor 1; CRF-R2, corticotropin-releasing factor receptor 2; SNS, Sini San; FD, functional dyspepsia.

A

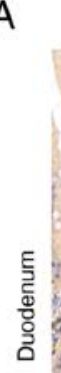

Control

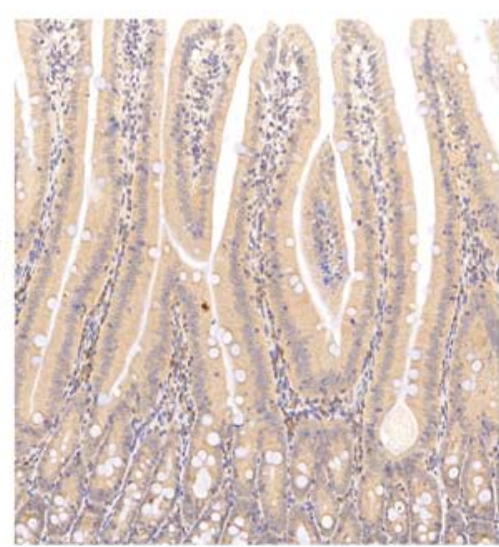

FD

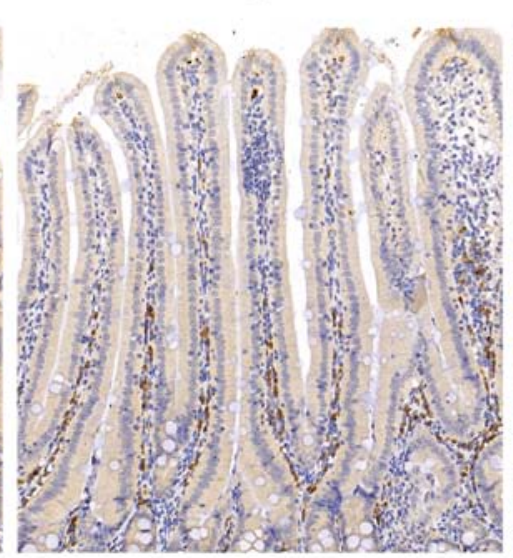

SNS

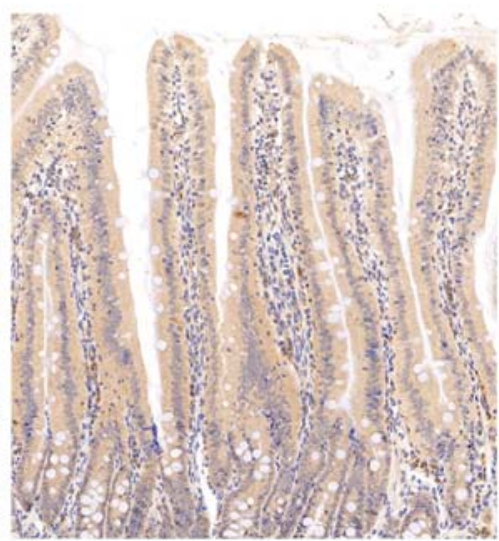

B

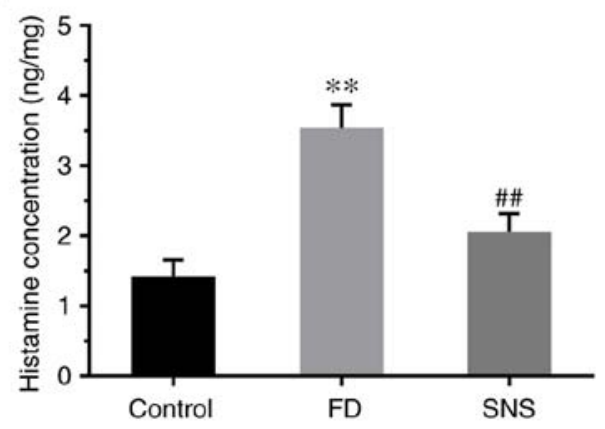

Figure 4. (A) Immunohistochemical staining of mast cells in the duodenum with an anti-tryptase antibody. Magnification, x100. (B) Concentration of histamine, as determined by ELISA. The expression of mast cell tryptase was elevated in the model group, and reduced following SNS treatment. The concentration of histamine was markedly higher in FD rats, and was decreased in the SNS group. $\mathrm{N}=4$ (A); $\mathrm{N}=6$ (B); ${ }^{* *} \mathrm{P}<0.01$ compared with the control group; ${ }^{\# \#} \mathrm{P}<0.01$ compared with the model group. SNS, Sini San; FD, functional dyspepsia.

integrity, which may be the underlying therapeutic mechanism of SNS in the treatment of FD.

The importance of low-grade inflammation in FD has been reported and confirmed in recent years (4). Systemic responses including an increased number of circulating lymphocytes, elevated expression levels of pro-inflammatory cytokines, and low-grade inflammation in the duodenum may promote the symptoms of FD (19). MCs are one of the dominant factors within the inflammatory infiltrate of the duodenum in FD, which has been previously confirmed $(34,35)$. In the present study, significant increases in the number of tryptase-positive MCs and the concentration of histamine were observed, suggesting that MC-associated pathways are activated in FD model rats. Findings of a previous study have suggested that there are interactive relationships between the gut epithelial barrier and intestinal inflammation (29). Degranulated MCs release MC tryptase that activates PAR-2 in the intestine, increasing paracellular permeability (10). The present study revealed that the mRNA expression levels of tryptase and PAR-2 were significantly increased in the FD group, indicating that the tryptase/PAR-2 pathway had been activated. Of note, it was also demonstrated for the first time that SNS suppressed the infiltration of MCs and inhibited the tryptase/PAR-2 pathway. Specifically, the influence of SNS on MC infiltration was assessed by IHC staining of tryptase. Results showed that the number of tryptase-positive MCs was elevated in the model group, and reduced following SNS treatment. The trend of histamine expression was the same, 

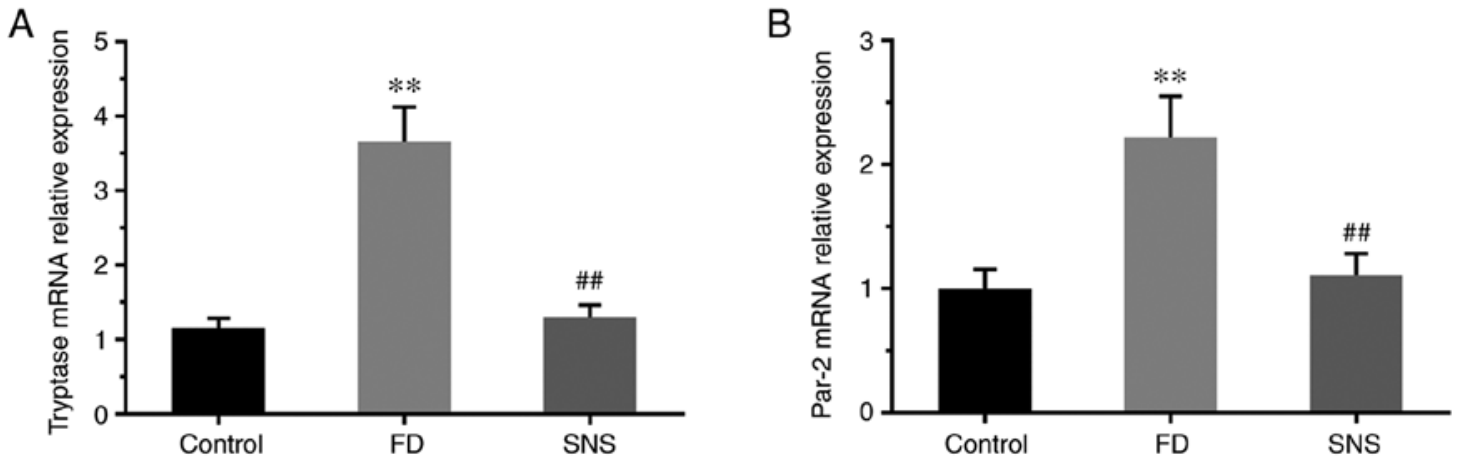

Figure 5. Relative mRNA expression levels of (A) tryptase and (B) PAR-2. mRNA expression levels of tryptase and PAR-2 were increased in the FD group, and SNS treatment reduced the expression of tryptase and PAR-2. $\mathrm{N}=6$ for each group. ${ }^{* *} \mathrm{P}<0.01$ compared with the control group; ${ }^{\# \#} \mathrm{P}<0.01$ compared with the model group. PAR-2, protease-activated receptor 2; FD, functional dyspepsia; SNS, Sini-san.
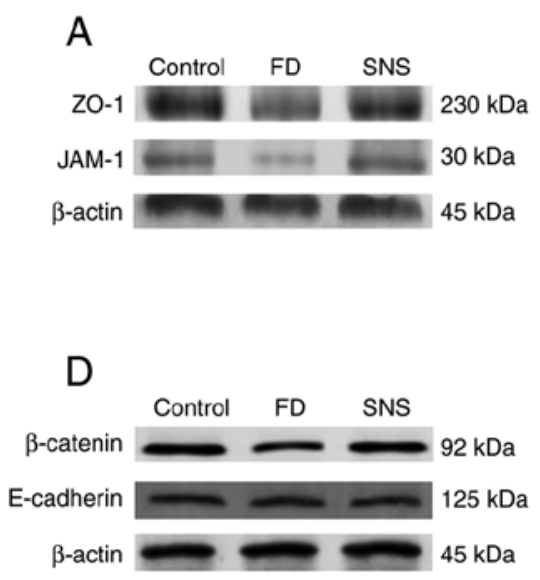

B
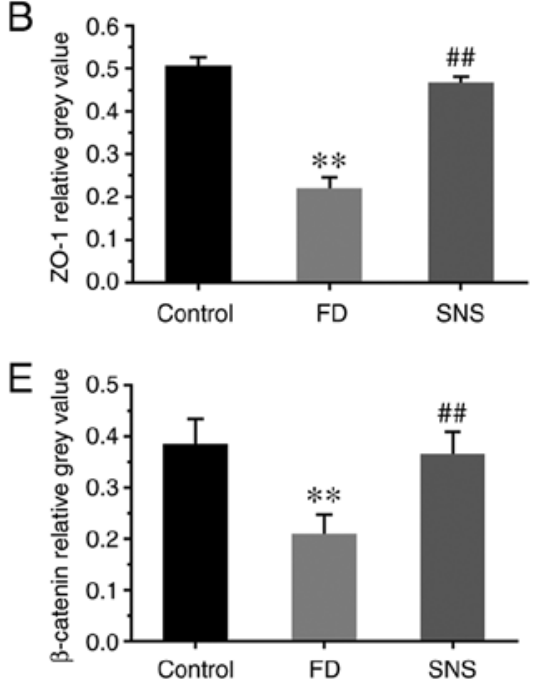
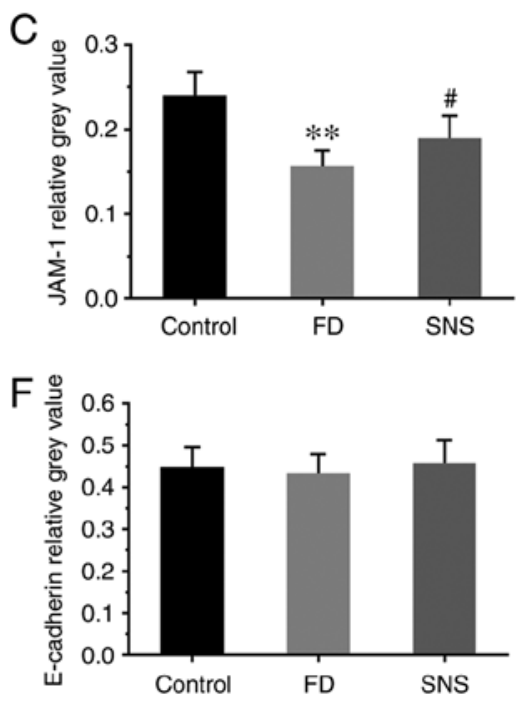

Figure 6. (A and D) Western blot analysis of ZO-1, JAM-1, $\beta$-catenin and E-cadherin expression in duodenal tissues. Relative grey values of ZO-1, JAM-1, $\beta$-catenin and E-cadherin expression levels. (B, C, E and F) Compared with the control group, the protein expression level of ZO-1, JAM-1 and $\beta$-catenin was markedly reduced in the model rats, and SNS treatment increased the expression levels of these three proteins. There was no significant difference in the protein expression level of E-cadherin between groups. $\mathrm{N}=6$ for each group. ${ }^{* *} \mathrm{P}<0.01$ compared with the control group; ${ }^{\#} \mathrm{P}<0.05$ and ${ }^{\# \#} \mathrm{P}<0.01$ compared with the model group. ZO-1, zona occludens protein-1; JAM-1, junctional adhesion molecule-1; SNS, Sini San; FD, functional dyspepsia.

whereas tryptase and PAR-2 mRNA expression levels were significantly reduced in the SNS group. These findings suggest that the MC tryptase/PAR-2 signaling pathway can be regulated by SNS. However, it remains unclear whether the central nervous system is involved in these pathological alterations.

It is widely acknowledged that pathological responses to gut-brain disorders are associated with FD (36). Anxiety and depression exist in a considerable proportion of patients with FD, and these individuals are prone to FD symptoms when exposed to stress (37). CRF is a key regulator of the brain-gut axis in response to stress, and is widely expressed in the central nervous system and the gastrointestinal tract. Various studies have shown that CRF signaling pathways affect inflammation and epithelial permeability in the intestine $(9,38)$, but as yet, it is not known whether CRF is an important regulatory factor in low-grade inflammation and mucus barrier integrity in the duodenum of FD patients. Another key finding of the present study was the identification of differences in the expression levels of CRF between the control and the FD groups, and that SNS significantly reduced these expression levels in both the central nervous system and the duodenum. It has also been reported that CRF regulates mucosal permeability via MCs (39). CRF in the brain and its periphery acts via its engagement with CRF-R1 and CRF-R2. Novel research suggests that CRF-R1 and CRF-R2 signaling is dynamic, but reaches a state of equilibrium in healthy individuals, thus can be utilized to determine functional changes in the gastrointestinal tract induced by stress (40). In the acute responses to immunologic and psychologic stress, CRF-R1 expressed on MCs as positive, global modulators of MC degranulation (41), while CRF-R2 plays a negative role in modulating the degranulation of MCs (42). In the present study, SNS treatment restored the balance of CRF1 and CRF2 signaling, suggesting that SNS is involved in the gut-brain interaction by regulating the CRF signaling pathway.

To conclude, the present study confirmed that decreased expression levels of AJ and TJ proteins, increased infiltration of MCs, and differential expression of CRF pathway components are associated with FD. Moreover, it was elucidated for the first time that the therapeutic effect of SNS on FD was achieved by 
restoring mucosal barrier integrity and suppressing low-grade inflammation in the duodenum, which was mediated via the CRF signaling pathway.

\section{Acknowledgements}

Not applicable.

\section{Funding}

The present study was supported by the National Natural Science Foundation of China (grant no. 81774215).

\section{Availability of data and materials}

The datasets used and analyzed during the current study are available from the corresponding author on reasonable request.

\section{Authors' contributions}

SZ and CZ contributed to the study concept and design; $\mathrm{CZ}$ and $\mathrm{JZ}$ performed the experiments and statistical analysis; $\mathrm{CZ}$ and LZ were involved in manuscript drafting and revision. All authors read and approved the final manuscript.

\section{Ethics approval and consent to participate}

The present study was performed in accordance with the Guide for the Care and Use of Laboratory Animals published by the National Institutes of Health, and was approved by the Animal Care and Use Committee of Beijing Institute of Traditional Chinese Medicine (no. 2018030101).

\section{Patient consent for publication}

Not applicable.

\section{Competing interests}

The authors declare that they have no competing interests.

\section{References}

1. Tack J, Talley NJ, Camilleri M, Holtmann G, Hu P, Malagelada JR and Stanghellini V: Functional gastroduodenal disorders Gastroenterology 130: 1466-1479, 2006.

2. Stanghellini V, Chan FK, Hasler WL, Malagelada JR, Suzuki H, Tack J and Talley NJ: Gastroduodenal disorders. Gastroenterology 150: 1380-1392, 2016.

3. Konturek SJ, Konturek PC, Pawlik T, Sliwowski Z, Ochmański W and Hahn EG: Duodenal mucosal protection by bicarbonate secretion and its mechanism. J Physiol Pharmacol 55 (Suppl 2): S5-S17, 2014

4. Vanheel H, Vicario M, Vanuytsel T, Van Oudenhove L, Martinez C, Keita ÅV, Pardon N, Santos J, Söderholm JD, Tack J and Farré R: Impaired duodenal mucosal integrity and low-grade inflammation in functional dyspepsia. Gut 63: 262-271, 2014.

5. Ishigami H, Matsumura T, Kasamatsu S, Hamanaka S, Taida T, Okimoto K, Saito K, Minemura S, Maruoka D, Nakagawa T, et al: Endoscopy-guided evaluation of duodenal mucosal permeability in functional dyspepsia. Clin Transl Gastroenterol 8: e83, 2017.

6. Koloski NA, Jones M, Kalantar J, Weltman M, Zaguirre J and Talley NJ: The brain-gut pathway in functional gastrointestinal disorders is bidirectional: A 12-year prospective population-based study. Gut 61: 1284-1290, 2012.
7. Stengel A and Taché YF: Activation of brain somatostatin signaling suppresses CRF receptor-mediated stress response. Front Neurosci 11: 231, 2017.

8. Hagiwara SI, Kaushal E, Paruthiyil S, Pasricha PJ, Hasdemir B and Bhargava A: Gastric corticotropin-releasing factor influences mast cell infiltration in a rat model of functional dyspepsia. PLoS One 13: e203704, 2018.

9. Wouters MM, Vicario M and Santos J: The role of mast cells in functional GI disorders. Gut 65: 155-168, 2016.

10. Jacob C, Yang PC, Darmoul D, Amadesi S, Saito T, Cottrell GS, Coelho AM, Singh P, Grady EF, Perdue M and Bunnett NW: Mast cell tryptase controls paracellular permeability of the intestine. Role of protease-activated receptor 2 and beta-arrestins. J Biol Chem 280: 31936-31948, 2005.

11. Feng He and Ling Qin: Clinical efficacy of Sini powder and western medicine in the treatment of mixed cold and heat functional dyspepsia. Acta Chin Med Pharmacol 42: 148-150, 2014.

12. Han YY and Wang HB: Clinical observation of modified sini powder in the treatment of 30 cases of functional dyspepsia with depression. Beijing J Trad Chin Med 30: 457-458, 2011.

13. Li Y, Sun Y, Ma X, Xue X, Zhang W, Wu Z, Ouyang Y, Chen J, Wang W, Guo S and Wang W: Effects of Sini san used alone and in combination with fluoxetine on central and peripheral 5-HT levels in a rat model of depression. J Tradit Chin Med 33: 674-681, 2013.

14. Chang X, Zhao L, Wang J, Lu X and Zhang S: Sini-san improves duodenal tight junction integrity in a rat model of functional dyspepsia. BMC Complement Altern Med 17: 432, 2017.

15. Chinese Pharmacopoeia Commission: Pharmacopoeia of the People's Republic of China. Vol 1. China Medical Science Press: 86-280, 2015.

16. Wu ZY, Zhang SS, Li PC, Lu XF, Wang ZF, Wang JJ and Li XL: Rat model of functional dyspepsia resulting from iodoacetamide-treated and tail-squeezed. Chin J Integrated Traditional Western Med Digestion 23: 462-466, 2015

17. Taché Y and Perdue MH: Role of peripheral CRF signalling pathways in stress-related alterations of gut motility and mucosal function. Neurogastroenterol Motil 16 (Suppl 1): S137-S142, 2004.

18. Taché Y, Martinez V, Million M and Rivier J: Corticotropinreleasing factor and the brain-gut motor response to stress. Can J Gastroenterol 13 (Suppl A): A18-A25, 1999.

19. Walker MM and Talley NJ: The role of duodenal inflammation in functional dyspepsia. J Clin Gastroenterol 51: 12-18, 2017.

20. Neilan NA, Garg UC, Schurman JV and Friesen CA: Intestinal permeability in children/adolescents with functional dyspepsia. BMC Res Notes 7: 275, 2014.

21. Larson D and Mitre E: Histamine release and surface CD200R1 staining as sensitive methods for assessing murine mast cell activation. J Immunol Methods 379: 15-22, 2012.

22. Fernández-Blanco JA, Hollenberg MD, Martinez V and Vergara P: PAR-2-mediated control of barrier function and motility differs between early and late phases of postinfectious gut dysfunction in the rat. Am J Physiol Gastrointest Liver Physiol 304: G390-G400, 2013.

23. Li S, Guan J, Ge M, Huang P, Lin Y and Gan X: Intestinal mucosal injury induced by tryptase-activated protease-activated receptor 2 requires $\beta$-arrestin-2 in vitro. Mol Med Rep 12: 7181-7187, 2015.

24. Camilleri M, Madsen K, Spiller R, Greenwood-Van Meerveld B and Verne GN: Intestinal barrier function in health and gastrointestinal disease. Neurogastroenterol Motil 24: 503-512, 2012.

25. Stremmel W, Staffer S, Schneider MJ, Gan-Schreier H, Wannhoff A, Stuhrmann N, Gauss A, Wolburg H, Mahringer A, Swidsinski A and Efferth T: Genetic mouse models with intestinal-specific tight junction deletion resemble an ulcerative colitis phenotype. J Crohns Colitis 11: 1247-1257, 2017.

26. Landy J, Ronde E, English N, Clark SK, Hart AL, Knight SC, Ciclitira PJ and Al-Hassi HO: Tight junctions in inflammatory bowel diseases and inflammatory bowel disease associated colorectal cancer. World J Gastroenterol 22: 3117-3126, 2016.

27. Martinez C, Vicario M, Ramos L, Lobo B, Mosquera JL, Alonso C, Sánchez A, Guilarte M, Antolín M, de Torres I, et al: The jejunum of diarrhea-predominant irritable bowel syndrome shows molecular alterations in the tight junction signaling pathway that are associated with mucosal pathobiology and clinical manifestations. Am J Gastroenterol 107: 736-746, 2012.

28. Boeckxstaens G, Camilleri M, Sifrim D, Houghton LA, Elsenbruch S, Lindberg G, Azpiroz F and Parkman HP: Fundamentals of neurogastroenterology: Physiology/motilitysensation. Gastroenterology: Feb. 18, 2016 (Epub ahead of print). 
29. Pastorelli L, De Salvo C, Mercado JR, Vecchi M and Pizarro TT: Central role of the gut epithelial barrier in the pathogenesis of chronic intestinal inflammation: Lessons learned from animal models and human genetics. Front Immunol 4: 280, 2013.

30. Hamada K, Shitara Y, Sekine S and Horie T: Zonula occludens-1 alterations and enhanced intestinal permeability in methotrexatetreated rats. Cancer Chemother Pharmacol 66: 1031-1038, 2010.

31. Naik UP and Eckfeld K: Junctional adhesion molecule 1 (JAM-1) J Biol Regul Homeost Agents 17: 341-347, 2003.

32. Lee HS, Kim DK, Kim YB and Lee KJ: Effect of acute stress on immune cell counts and the expression of tight junction proteins in the duodenal mucosa of rats. Gut Liver 7: 190-196, 2013.

33. Tian X, Liu Z, Niu B, Zhang J, Tan TK, Lee SR, Zhao Y, Harris DC, Zheng G: E-cadherin/ $\beta$-catenin complex and the epithelial barrier. J Biomed Biotechnol 2011: 567305, 2011.

34. Yuan HP, Li Z, Zhang Y, Li XP, Li FK and Li YQ: Anxiety and depression are associated with increased counts and degranulation of duodenal mast cells in functional dyspepsia. Int J Clin Exp Med 8: 8010-8014, 2015.

35. Vanheel H, Vicario M, Boesmans W, Vanuytsel $T$, Salvo-Romero E, Tack J and Farré R: Activation of eosinophils and mast cells in functional dyspepsia: An ultrastructural evaluation. Sci Rep 8: 5383, 2018.

36. Powell N, Walker MM and Talley NJ: The mucosal immune system: Master regulator of bidirectional gut-brain communications. Nat Rev Gastroenterol Hepatol 14: 143-159, 2017.

37. De la Roca-Chiapas JM, Solís-Ortiz S, Fajardo-Araujo M, Sosa M, Córdova-Fraga T and Rosa-Zarate A: Stress profile, coping style, anxiety, depression, and gastric emptying as predictors of functional dyspepsia: A case-control study. J Psychosom Res 68: 73-81, 2010.
38. Overman EL, Rivier JE and Moeser AJ: CRF induces intestinal epithelial barrier injury via the release of mast cell proteases and TNF- $\alpha$. PLoS One 7: e39935, 2012.

39. Wallon C, Yang PC, Keita AV, Ericson AC, McKay DM, Sherman PM, Perdue MH and Söderholm JD: Corticotropinreleasing hormone $(\mathrm{CRH})$ regulates macromolecular permeability via mast cells in normal human colonic biopsies in vitro. Gut 57: 50-58, 2008

40. Nozu T and Okumura T: Corticotropin-releasing factor receptor type 1 and type 2 interaction in irritable bowel syndrome. J Gastroenterol 50: 819-830, 2015.

41. Ayyadurai S, Gibson AJ, D'Costa S, Overman EL, Sommerville LJ, Poopal AC, Mackey E, Li Y and Moeser AJ: Frontline science: Corticotropin-releasing factor receptor subtype 1 is a critical modulator of mast cell degranulation and stress-induced pathophysiology. J Leukoc Biol 102: 1299-1312, 2017.

42. D'Costa S, Ayyadurai S, Gibson AJ, Mackey E, Rajput M, Sommerville LJ, Wilson N, Li Y, Kubat E, Kumar A, et al: Mast cell corticotropin-releasing factor subtype 2 suppresses mast cell degranulation and limits the severity of anaphylaxis and stress-induced intestinal permeability. J Allergy Clin Immunol 143: 1865-1877, 2019.

This work is licensed under a Creative Commons Attribution-NonCommercial-NoDerivatives 4.0 International (CC BY-NC-ND 4.0) License. 